\title{
Turbulent Flow in an Annular Channel of Variable Cross-Section
}

\author{
Vladimir Trifonov ${ }^{1}$, Alexander Reshmin ${ }^{1}$, Sergei Teplovodskii ${ }^{1}$ \\ ${ }^{1}$ Institute of Mechanics Lomonosov Moscow State \\ 1 Michurinskii prospect, Moscow, Russia \\ trifonov@imec.msu.ru; alexreshmin@rambler.ru; tipa49@mail.ru
}

\begin{abstract}
The flow in an annular channel of variable cross-section is studied numerically. The flow is assumed to be continuous, so only small angles of inclination of the channel walls are considered. For the Reynolds numbers in the range 3000-10000, the velocity profiles, turbulence energies, and Reynolds stresses and the characteristics of turbulent heat transfer determined by them are calculated. Calculations show a strong influence of the opening angle of the annular channel on the turbulent flow characteristics. An increase in the intensity of turbulence in the case of heat supply to the wall leads to an increase in the heat transfer characteristics. When using such a channel in a heat exchanger, it is possible to increase the Nusselt number and the Reynolds analogy factor without using artificial flow turbulization in comparison with a channel of constant cross-section at the same Reynolds numbers, that is, to increase the heat exchange without significantly increasing the channel resistance. The calculations are carried out using a three-parameter differential turbulence model supplemented with equations for turbulent heat transfer.
\end{abstract}

Keywords: annular channel, heat transfer rate, Reynolds analogy factor.

\section{Introduction}

An experimental study of the characteristics of air flow in the outlet section of a circular conical diffuser with an angle of inclination of the generatrix of $\sim 0.3 \mathrm{deg}$. and a length of $\sim 70$ inlet diameters at $600<\operatorname{Re}<3000$ showed a strong effect of channel expansion on the flow regime [1,2]. In contrast to a pipe, where, at a value of $\operatorname{Re}<2000$, only a laminar flow is realized as a steady-state flow, a turbulent flow was formed at the outlet of the diffuser under study at Re values greater than $\sim 1000$. The formation of a turbulent flow was confirmed by the results of direct measurements of the Reynolds stresses. It was noted that even at such a small opening angle of the channel, the measured profiles of turbulent stresses in amplitude were almost twice the corresponding values in a round tube at the same Reynolds numbers. Calculations of the flow parameters in the diffuser showed that the measured velocity and turbulent stresses in the outlet section of the diffuser are well described by a three-parameter differential model of shear turbulence [3].

The published results of experimental studies of a flow with heat transfer in a flat converging channel [4] showed that turbulence in it is suppressed, and there are regimes in which almost complete laminarization occurs. Numerical analysis of these results using a three-parameter differential model, supplemented by equations for turbulent heat transfer [5], also showed the applicability of the specified calculation model [6].

The discovered influence of the channel shape on the turbulence characteristics aroused interest in using this effect in heat exchange devices. Turbulent and thermal characteristics of flat and round expanding channels were numerically investigated, a scheme of a plate heat exchanger with diffuser channels was proposed, and its characteristics were calculated [7]. It is shown that the Stanton number over the entire length and the heat transfer coefficient over most of the length in a heat exchanger with diffuser channels exceed the corresponding values for a heat exchanger with channels of constant cross section. The values of the turbulence intensity and shear stress obtained in the calculations significantly increase in the diffuser, which leads to the intensification of heat transfer. Static pressure losses in diffuser channels at small (up to $0.8^{\circ}$ ) opening angles are less than static pressure losses in channels of constant cross-section at the same Reynolds numbers [7].

In the present work, using a three-parameter differential turbulence model, we study numerically turbulent characteristics of the flow in an annular channel. Such channels are widely used in tubular heat exchangers (tube-in-tube heat exchanger). Conical walled annular channels with different angles of inclination will be considered. 
It should be noted that the turbulence model $[3,5]$ has been comprehensively tested in a wide class of boundary layer problems. The testing of the turbulence model [6] and the experimentally confirmed results of calculating the flow characteristics in a circular diffuser [1,2] give grounds for using it in this work.

\section{Statement of the problem}

\subsection{Three-Parameter Model of Shear Turbulence}

The model developed by V. G. Lushchik, A. A. Pavelyev, and A. E. Yakubenko for analyzing the development of grid turbulence was then expanded for various non-equilibrium turbulent flows, in which it is necessary to take into account the diffusion terms in the transport equations. This model includes transport equations for three parameters, namely: turbulent shear stress, turbulence energy, and the ratio of turbulent energy to turbulence scale to the second degree [3].

It should be emphasized that the model constants obtained as a result of the analysis of three reference flows (flow behind the grid without an average velocity gradient; flow behind the grid with an average velocity gradient and developed turbulent flow in the pipe) did not change when the model was applied to other flows. For turbulent flows of variable density, the analysis of the effect of deformation on the characteristics of turbulence is carried out.

This model has been used to calculate a wide class of turbulent flows in boundary layers and channels. The calculations were carried out for both incompressible and compressible flows. In particular, calculations using this model were carried out for the following flows.

- Boundary layers with injection and suction.

- Boundary layers with positive and negative pressure gradients.

- Boundary layers along the length of the transition to turbulence at high intensity of external disturbances.

- A new class of developing channel flows with inhomogeneous velocity profiles and small-scale turbulence in the initial section, etc.

In all cases, satisfactory agreement with the experimental results was noted. The results calculated using this model are compared with the results of direct numerical simulation of asymmetric turbulent flow between permeable plates, through one of which gas is injected and through the other is sucked out. The results of direct numerical simulation of such a flow are in very good agreement with the results of calculations using the model described above.

The developed turbulence model, supplemented by the transfer equation for a turbulent heat flux [5], can be successfully used to calculate turbulent flows with heat exchange in channels of variable cross-section.

\subsection{Features of the Flow in the Annular Channel}

Compared to flows in flat and circular channels, the flow in annular channels can be more diverse. The hydraulic diameter, calculated by the formula $\mathrm{D}_{\mathrm{h}}=4 \mathrm{~F} / \mathrm{P}$ (where $\mathrm{F}$ is the channel cross-sectional area, and $\mathrm{P}$ is the wetted perimeter) is equal to two heights $\mathrm{H}$ for a flat channel, diameter $\mathrm{D}$ for a round channel, and the difference $\left(\mathrm{D}_{2}-\mathrm{D}_{1}\right)$ of the outer and inner diameters for annular channel. Reynolds number $\operatorname{Re}=\rho U D_{h} / \eta$, where $\rho$ is density, $U$ is average velocity, $\eta$ is dynamic viscosity. If the flow rate $\mathrm{G}=\mathrm{F} \rho \mathrm{U}$ and the temperature along the channel length do not change, then the Reynolds number turns out to be constant for a flat channel, inversely proportional to the diameter $\operatorname{Re} \sim 1 / \mathrm{D}$ for a round channel and inversely proportional to the sum of the inner and outer diameters $\operatorname{Re} \sim 1 /\left(D_{1}+D_{2}\right)$ for the annular channel. It can be seen that in a flat channel, a change in the opening angle unambiguously changes the average speed, and in a round channel, the average speed and Reynolds number. In an annular channel, the opening angle depends on the angles of inclination of the generatrices of the conical walls and does not determine the change in the Reynolds number. So at any opening angles, the Reynolds number can remain constant if the angles of inclination of the walls are equal and opposite in sign $\alpha_{1}=-\alpha_{2}$ (Fig. 1). On the other hand, as the Reynolds number changes, the average flow rate can remain constant.

This nature of the flow in an annular channel allows us to study the dependence of the turbulent and thermal characteristics of the flow on both the Reynolds number and the opening angles in their various combinations.

\subsection{Calculation Scheme}

The problem was solved in the following setting (Fig. 1). A developed turbulent flow is established at the inlet section of length $L_{1}$ with constant wall diameters and channel height $h_{1}$. This is followed by the main section with a change in the inner and outer diameters of the channel length $\mathrm{L}_{\mathrm{d}}$. Behind it there is an outlet section of constant cross-section with diameters equal to the diameters at the outlet from the main section, where a flow corresponding to the flow parameters can be established. $\mathrm{X}=\mathrm{x} / \mathrm{L}_{\mathrm{d}}$ was chosen as the longitudinal dimensionless coordinate, where $\mathrm{x}$ is the longitudinal coordinate with zero at the beginning of the main channel section. The opening angle of the channel is equal to the difference in the angles 
of inclination of the outer and inner walls of the channel. The opening angle is considered positive if it increases the channel height, and negative if it decreases it.

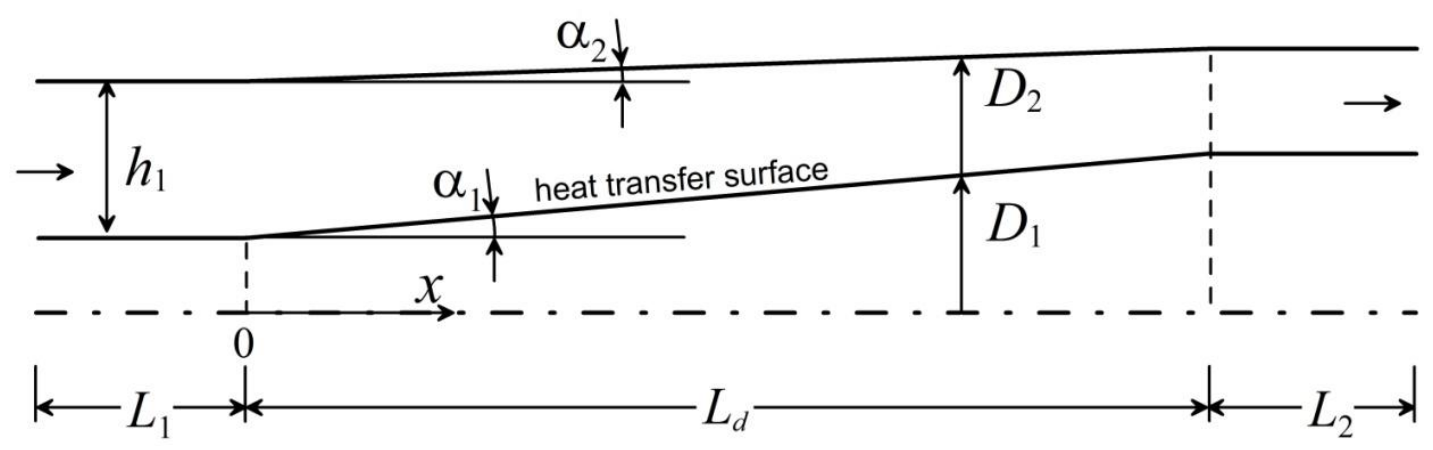

Fig.1. Calculation scheme.

When conducting thermal calculations, the heat flux is set on the inner wall of the main section of the channel and is assumed constant along its length and small in magnitude so that the thermophysical and transport properties of the heat carrier could be considered constant.

As a result, the local values of the Nusselt number $\mathrm{Nu}$, the drag coefficient $\xi$, and the Reynolds analogy factor Raf were obtained, defined as

$$
\mathrm{Nu}=\frac{D_{h} q_{w}}{\lambda\left(T_{w}-\bar{T}\right)}, \quad \xi_{w}=8 \frac{\tau_{w}}{\rho U^{2}}, \quad \xi=\left(\xi_{w 1} D_{1}+\xi_{w 2} D_{2}\right) /\left(D_{1}+D_{2}\right), \quad \operatorname{Raf}=\frac{8}{\xi} \frac{\mathrm{Nu}}{\operatorname{Re} \operatorname{Pr}}
$$

Here $\mathrm{T}_{\mathrm{w}}$ is the wall temperature, $\lambda$ is the thermal conductivity, $\bar{T}$ is the mass average temperature, $\mathrm{U}$ is the average velocity, Pr is the Prandtl number, $\tau_{w}=(\eta \partial u / \partial y)_{w}, q_{w}=-(\lambda \partial T / \partial y)_{w}$. The subscript $w$ marks the values of the parameters on the channel wall ( $\mathrm{w}_{1}$ - on the inner wall, $\mathrm{w}_{2}$ - on the outer wall), $\mathrm{y}$ is the direction normal to the wall.

The calculations also obtained dimensionless velocity profiles, turbulence intensity, and turbulent friction.

The following values are the parameters of the problem:

- Reynolds number Re;

- opening angle $\alpha=\alpha_{2}-\alpha_{1}$.

Below are the results of the numerical study of turbulent flow characteristics and their effect on the intensification of heat transfer.

\section{Turbulent and thermal characteristics of the flow}

\subsection{Influence of the opening angle on turbulent characteristics at $\operatorname{Re}=$ const $\left(\alpha_{1}=-\alpha_{2}\right)$}

The peculiarity of the annular channel is that the Reynolds number can remain constant during both expansion and contraction of the channel in height. If the walls of the channel are inclined in different directions at the same angles in absolute value, then the sum of the outer and inner diameters, and hence the Reynolds number, remain constant. In this case, it is possible to determine the effect of flow acceleration on flow characteristics. Fig. 2 shows the change along the channel length of the maximum turbulence intensity $e=\sqrt{E_{\max }} / U \quad\left(E=0.5 \sum_{i}<u_{i}^{\prime 2}>\right)$ depending on the opening angle for Re $=$ 3000 and $\operatorname{Re}=10000$. 

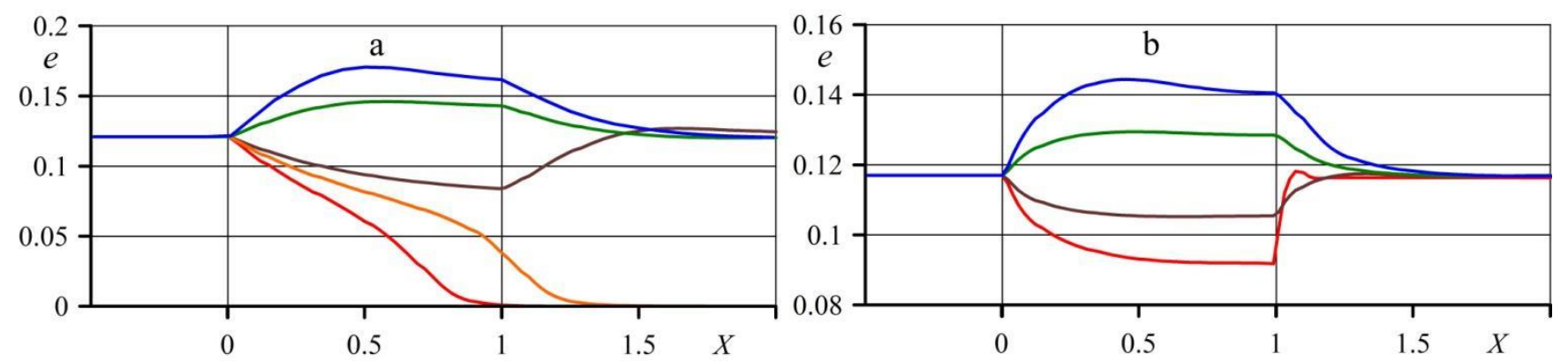

Fig. 2. Change the maximum intensity of turbulence $e^{e=\sqrt{E_{\max }}} / U$ of the air flow along the length of $X=x / L_{d}$ channel depending on the opening angle $\alpha$ at $\operatorname{Re}=3000$ (a) and $\operatorname{Re}=10000$ (b).

$\alpha$ : blue $1^{\circ}$, green $0.5^{\circ}$, brown $-0.5^{\circ}$, orange $-0.7^{\circ}$, red $-1^{\circ}$.

It is seen that during the flow in the expanding channel, the turbulence intensity increases, while in the converging channel it decreases. In the outlet section of a constant cross-section, the turbulent characteristics are restored if the turbulence level does not decrease below the critical one. At the Reynolds number $\mathrm{Re}=3000$, if the angle of contraction in absolute value exceeds 0.7 degrees, the turbulence completely degenerates. Degeneration of turbulence can occur in the expansion section, or already at the outlet section of constant cross-section, if e after the expansion section does not exceed 0.04.

The change in the intensity of turbulence during heat exchange causes corresponding changes in the Nusselt number. Changes in the hydraulic resistance are much less pronounced. Fig. 3 (a) shows the dependences of the Nusselt number for a constant heat flow set on the inner wall of the channel and $\mathrm{Re}=5000$ over the entire length of the channel. With a positive opening angle, the Nusselt number increases, and then, in the output part of the channel, the thermal characteristics are restored, if there is no complete degeneration of turbulence. It can be seen that at the angle $\alpha=-1^{\circ}$, the Nusselt number in the output section comes to its laminar value.
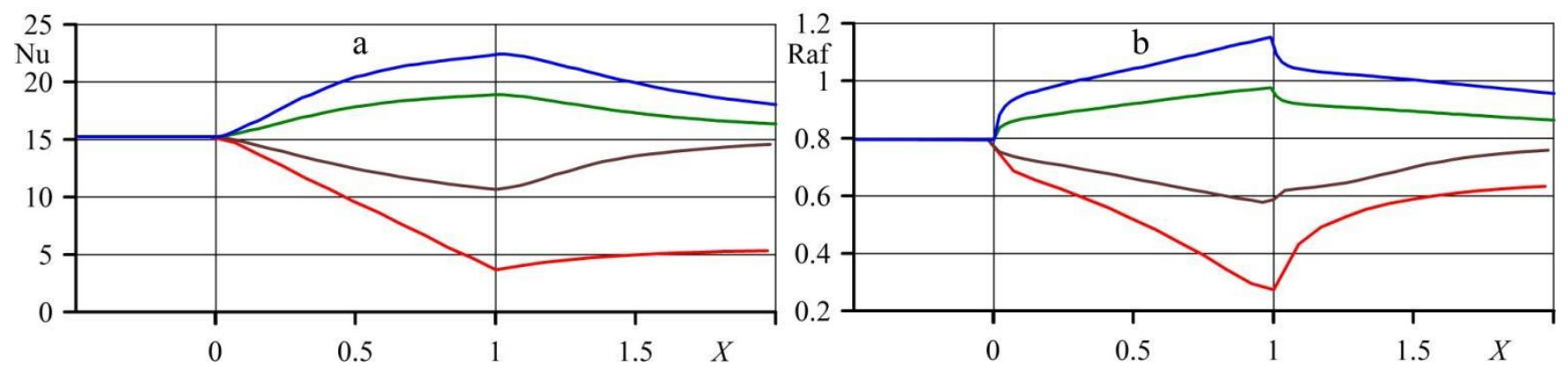

Fig. 3. Change in the Nusselt number Nu (a) and the Reynolds analogy factor Raf (b) of the air flow along the length $X=x / L_{d}$ of the channel depending on the opening angle $\alpha$ at $\mathrm{Re}=5000$. $\alpha$ : blue $1^{\circ}$, green $0.5^{\circ}$, brown $-0.5^{\circ}$, red $-1^{\circ}$.

Fig. 3 (b) shows the variation along the channel length of the Reynolds analogy factor for different values of the angle $\alpha$. It is seen that in the expanding channel the increase in the Nusselt number exceeds the change in the drag coefficient. 


\subsection{Flow characteristics with a constant diameter of the outer channel wall}

The turbulent and heat exchange characteristics of the flow in an expanding annular channel with a constant outer diameter are considered. In this case, the diameter of the inner wall decreases, and the Reynolds number in the expanding channel will increase. Fig. 4 shows the extreme values of the turbulence intensity and Reynolds stresses $\tau=-\left\langle u^{\prime} v^{\prime}\right\rangle / U^{2}$ along the channel length at different opening angles.
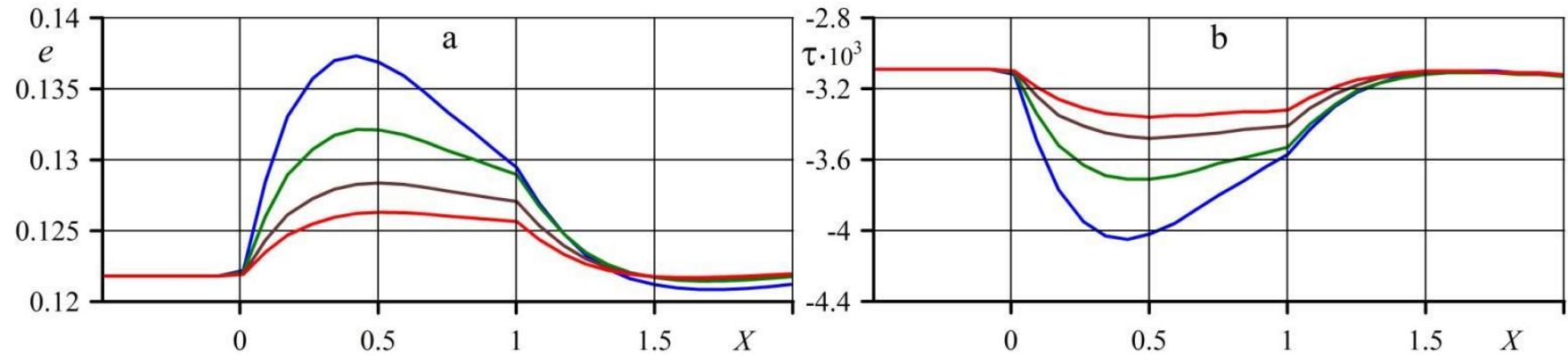

Fig 4. Change the extreme values of the turbulence intensity (a) and Reynolds stresses (b) along the channel length at different opening angles $\alpha$ with a constant outer channel diameter at $\operatorname{Re}=5000$ at the beginning of the flow. $\alpha$ : blue $0.8^{\circ}$, green $0.5^{\circ}$, brown $0.3^{\circ}$, red $0.2^{\circ}$.

The steady-state values of these parameters for the initial and outlet sections, where the channel wall diameters are constant, do not differ greatly. Therefore, it should be assumed that the noticeable deviations of these values from the steadystate values are determined by the geometry of the channel and are caused by the deceleration of the flow during expansion. Similar changes in the intensity of turbulence and Reynolds stresses during expansion are observed both in a flat channel at a constant Reynolds number and in a round channel, where the Reynolds number decreases.

With a constant heat flow on the inner wall of the channel, the Nusselt number depends on the Reynolds number and increases with the latter. In order to reveal the influence on the Nusselt number only of the channel geometry in these circumstances, in Fig. 5 the heat transfer characteristics are presented in the form of relative values of the Nusselt number $\mathrm{Nu} / \mathrm{Nu}_{0}$ and the Reynolds analogy factor $R a f / \mathrm{Raf}_{0}$. The Nusselt number $\mathrm{Nu}_{0}$ and the Reynolds analogy factor $\mathrm{Raf}_{0}$ are calculated for a developed turbulent flow in an annular channel of constant cross section at the Reynolds number and wall diameters corresponding to local values.
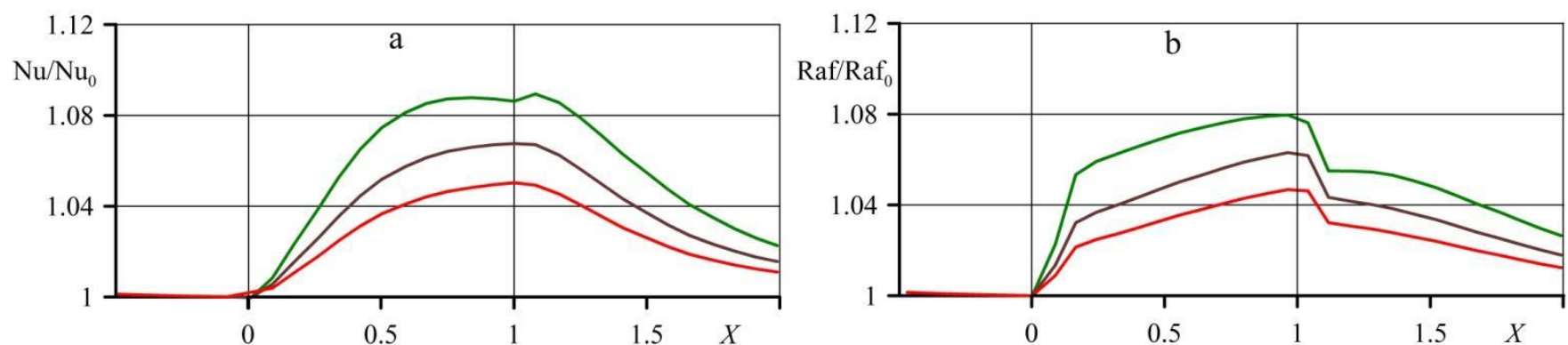

Fig 5. Change along the length of the relative values of the Nusselt number and the Reynolds analogy factor from the opening angle $\alpha$ at a constant outer channel diameter at $\operatorname{Re}=5000$ at the beginning of the flow.

$\alpha$ : green $0.5^{\circ}$, brown $0.3^{\circ}$, red $0.2^{\circ}$. 


\section{Conclusion}

Using a three-parameter differential turbulence model supplemented by the transfer equation for a turbulent heat flux, a numerical study of the flow and heat transfer in annular channels with different degrees of expansion is carried out. Air under normal conditions was considered as a heating medium.

It is shown that in an annular expanding channel with all the considered changes in the opening angle and Reynolds numbers, the main characteristics of heat transfer - the Nusselt number and the Reynolds analogy factor - turn out to be higher than in a channel of constant cross-section with the same Reynolds number. This excess increases with an increase in the opening angle.

Intensification of heat transfer in the expanding annular channel is achieved without a noticeable increase in the coefficient of friction. This is the fundamental difference between the considered method for intensifying heat transfer from many known methods, where an increase in heat transfer is achieved at the cost of a significant increase in hydraulic losses.

\section{Acknowledgements}

The study was carried out under the financial support of the Russian Science Foundation, grant no. 20-19-00404.

\section{References}

[1] Reshmin, A.I., Teplovodskii, S.K. \& Trifonov, V.V. "Turbulent flow in a circular separationless diffuser at Reynolds numbers smaller than 2000," Fluid Dyn46, 278-285, 2011.

[2] Reshmin A.I., Trifonov V.V., Teplovodskii S.Kh. "Turbulent flow in a conical diffuser with a small divergence angle at Reynolds numbers less than 2000," Proceedings of the ASME 2014 4th Joint US-European Fluids Engineering Division Summer Meeting, FEDSM2014, American Society of Mechanical Engineers, United States Chicago, Illinois, V. 21597, pp. 1-9.

[3] Lushchik V. G., Pavel'ev A. A., Yakubenko A. E.“Three-parameter model of shear turbulence,” Fluid Dyn 13, 350350, 1978.

[4] Tanaka H., Kawamura H., Tateno A., Hatamiya S. "Effect of Laminarization and Retransition on Heat Transfer for Low Reynolds Number Flow Through a Converging to Constant Area Duct," J. Heat Transfer, 104(2), 363-371, 1982.

[5] Lushchik V. G., Pavel'ev A. A., Yakubenko A. E."Three-parameter model of shear turbulence: heat transfer calculations," Fluid Dyn 21, 200-211, 1986.

[6] Lushchik, V.G., Makarova, M.S. \& Reshmin, A.I. Laminarization of Flow with Heat Transfer in a Plane Channel with a Confuser. Fluid Dyn54, 67-76, 2019.

[7] Lushchik, V.G., Makarova, M.S. \& Reshmin, A.I. Plate Heat Exchanger with Diffuser Channels. High Temp58,352$359,2020$. 